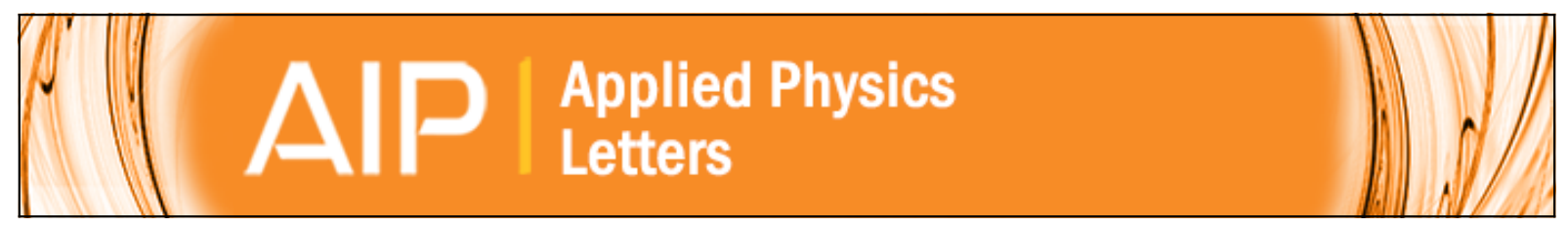

\title{
Electron and trap dynamics in As-ion-implanted and annealed GaAs
}

L. Giniũnas, R. Danielius, H. H. Tan, C. Jagadish, R. Adomavičius, and A. Krotkus

Citation: Applied Physics Letters 78, 1667 (2001); doi: 10.1063/1.1356727

View online: http://dx.doi.org/10.1063/1.1356727

View Table of Contents: http://scitation.aip.org/content/aip/journal/apl/78/12?ver=pdfcov

Published by the AIP Publishing

\section{Articles you may be interested in}

Transient reflectivity as a probe of ultrafast carrier dynamics in semiconductors: A revised model for lowtemperature grown GaAs

J. Appl. Phys. 116, 073506 (2014); 10.1063/1.4892868

Modeling of carrier lifetimes in uniaxially strained GaAs

J. Appl. Phys. 111, 103704 (2012); 10.1063/1.4717246

Deep level photothermal spectroscopy: Physical principles and applications to semi-insulating GaAs band-gap multiple trap states

J. Appl. Phys. 103, 043704 (2008); 10.1063/1.2842401

Subpicosecond carrier dynamics in low-temperature grown GaAs on Si substrates

Appl. Phys. Lett. 75, 2575 (1999); 10.1063/1.125082

High-carrier-density electron dynamics in low-temperature-grown GaAs

Appl. Phys. Lett. 70, 3245 (1997); 10.1063/1.119138

\section{Model PS-100}

Tabletop Cryogenic

Probe Station

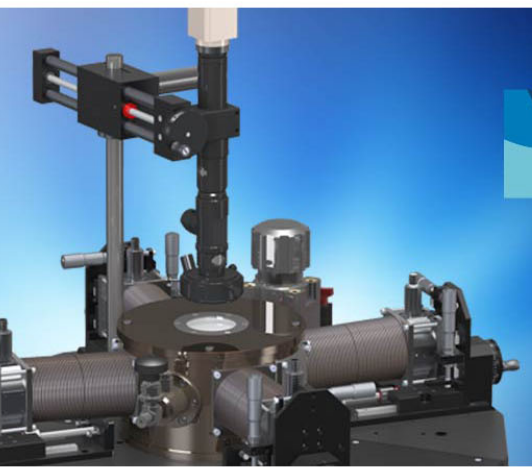

\section{Lake Shore} CRYOTRONICS

An affordable solution for a wide range of research 


\title{
Electron and trap dynamics in As-ion-implanted and annealed GaAs
}

\author{
L. Giniũnas and R. Danielius \\ Laser Research Center, Vilnius University, Sauletekio al. 10, Vilnius, 2040, Lithuania
}

H. H. Tan and C. Jagadish

Department of Electronic Materials Engineering, Research School of Physical Sciences and Engineering, The Australian National University, Canberra, ACT 0200 Australia

\author{
R. Adomavičius and A. Krotkus ${ }^{\text {a) }}$ \\ Semiconductor Physics Institute, A. Goštauto 11, Vilnius, 2600, Lithuania
}

(Received 1 June 2000; accepted for publication 29 January 2001)

\begin{abstract}
The ultrafast dynamics of As-ion-implanted and annealed GaAs is investigated using transmission pump-probe measurements. Carrier recombination time was found to increase from 4 to $40 \mathrm{ps}$ with increasing annealing temperature. At lower annealing temperatures, the transmitted optical signal is dominated by induced absorption and at higher annealing temperatures this effect is replaced by induced transparency. (C) 2001 American Institute of Physics. [DOI: 10.1063/1.1356727]
\end{abstract}

Semiconductors with ultrashort carrier lifetimes, good optical responsivity, and low conductivity are essential for various ultrafast optical and optoelectronic applications including saturable semiconductor absorber mirrors, ultrafast detectors and switches, and terahertz radiation sources. Recently, nonstoichiometric arsenic-rich GaAs grown by molecular-beam epitaxy at low substrate temperatures [lowtemperature-grown (LTG) GaAs] was the subject of intensive studies aiming at these applications. ${ }^{1}$ It exhibited nearly ideal electrical and optical properties for ultrafast applications. Recently a new class of arsenic-rich material, As-ionimplanted GaAs (GaAs:As) has emerged as a potential alternative to LTG GaAs. ${ }^{2,3}$ The structural and electrical characteristics of LTG GaAs and GaAs:As have been shown to be quite similar, ${ }^{4}$ and the ultrafast optical response of GaAs:As has been also documented. ${ }^{3,5,6}$

As a rule, the ultrafast response in a semiconductor material is measured by exciting nonequilibrium carriers with a stronger part of a femtosecond laser pulse and sampling the change of the optical characteristics by a second, weaker part of the same pulse. Moderate intensity band-to-band excitation is used in the majority of the experiments, therefore, the established reflectivity or the photoluminescence dynamics yields information solely on the free-electron trapping times in the material. However, as long as the trapped photoelectrons do not recombine with holes from the valence band, the material recovery is incomplete. It has been established that the recombination times in LTG GaAs can be as long as a few tens of picoseconds, ${ }^{7,8}$ the values of this critical device performance parameter in GaAs:As have not yet been investigated.

In this letter, we present a study of carrier recombination in As-ion-implanted and annealed (at temperatures from 500 to $700{ }^{\circ} \mathrm{C}$ ) GaAs layers, which were epitaxially lifted off (ELO) after implantation and annealing. The electron trapping times in these layers have been investigated by using femtosecond Ti:sapphire laser pulses and the temporally re-

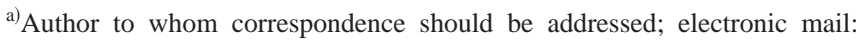
krotkus@uj.pfi.lt
}

solved photoluminescence (PL) used in a previous work ${ }^{9}$ and were found to vary from 100 fs to 5 ps. Here, we employed powerful picosecond laser pulses with a wavelength longer than the absorption edge of GaAs and a pump-and-probe technique, which allowed us to monitor simultaneously the photoinduced changes in the sample absorption and refractivity. It has been found that the carrier recombination time increases with increasing annealing temperatures from 4 to 40 ps. Also, depending on the annealing conditions, the increased occupancy of the band-gap states can lead to either photoinduced absorption or absorption bleaching.

$2-\mu \mathrm{m}$-thick GaAs layers with a 50-nm-thick AlAs liftoff layer were grown on GaAs substrates by the low-pressure metal-organic chemical-vapor deposition (MOCVD) technique at a substrate temperature of $700{ }^{\circ} \mathrm{C} .2 \mathrm{MeV}$ As ions were implanted at a dose of $1 \times 10^{16} \mathrm{~cm}^{-2}$ using the Australian National University tandem accelerator. During implantation the wafers were tilted $7^{\circ}$ off the beam axis to minimize channeling effects. The ion dose rate was $8 \mathrm{nA} / \mathrm{cm}^{2}$, and the nominal sample temperature was room temperature. After implantation, the samples were annealed for $20 \mathrm{~min}$ at different temperatures under arsine ambient in the MOCVD reactor.

The experimental setup was based on a mode-locked $\mathrm{Nd}$ :phosphate glass laser $(1.7 \mathrm{ps}, 10 \mathrm{~Hz}, 1.054 \mu \mathrm{m})$. The maximal single pulse energy used was $15 \mu \mathrm{J}$ with a pump pulse up to 100 times stronger than the probe pulse. The ELO GaAs:As sample was placed at 2 Rayleigh lengths before the waist of the focused laser beams where the Gaussian beam diameter was $340 \mu \mathrm{m}$. The part of the pump pulse absorbed in the sample via two-photon absorption (TPA), and/or absorption at the defects, creates nonequilibrium carriers that induce a refractive-index gradient and modify the occupation of the band-gap states in the sample. These changes were monitored by measuring the overall probe pulse energy transmitted through the sample (open aperture, or OA signal) and the energy of the central part of the probe beam (closed aperture, or CA signal). This type of experiment is similar to the dynamic $z$-scan technique. ${ }^{10}$ The results of the $Z$ scan are affected by the contributions of both 

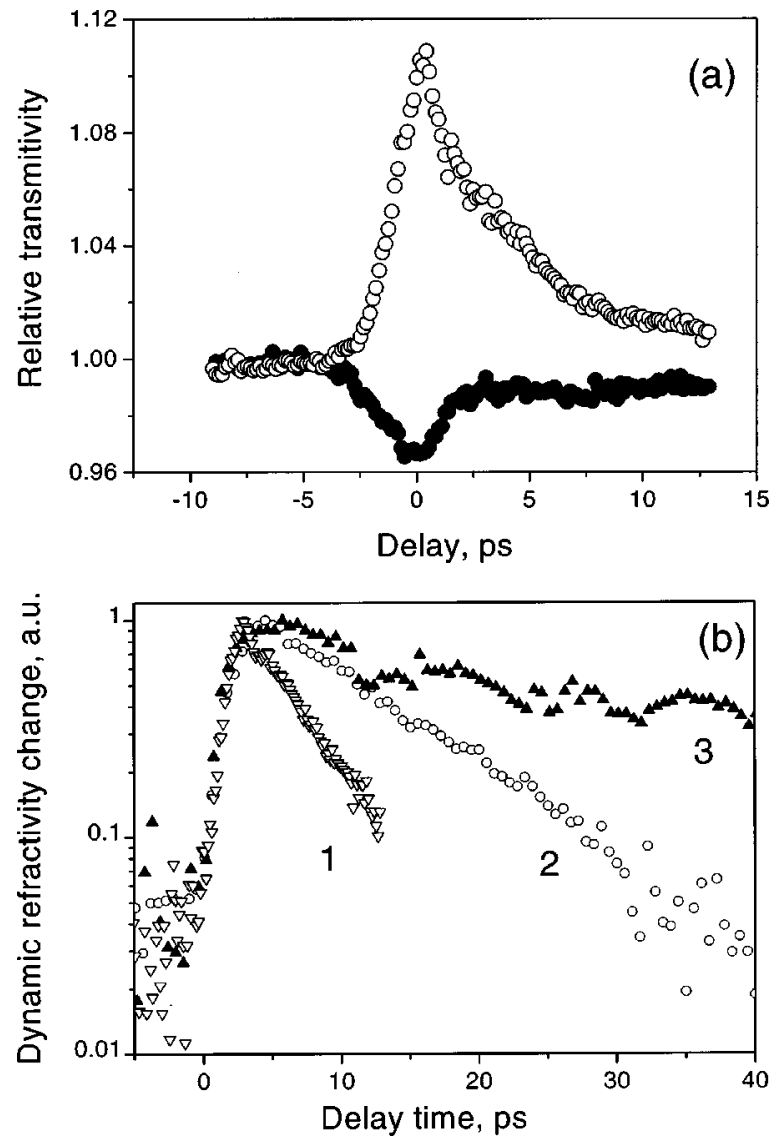

FIG. 1. (a) Pump pulse induced change in transmitivity of the probe pulse measured on a GaAs:As sample annealed at $500{ }^{\circ} \mathrm{C}$. Full circles, CA signal; and open circles, OA signal. (b) Dynamics of the ratio of CA and OA traces measured on samples annealed at $500{ }^{\circ} \mathrm{C}(1), 520^{\circ} \mathrm{C}(2)$, and $580{ }^{\circ} \mathrm{C}(3)$.

nonlinear optical refraction and absorption. In stationary $Z$-scan measurements the later contribution is determined from the OA signal whereas the first is found after dividing the CA trace by the OA Z-scan trace. We have applied a similar procedure by interpreting our results of the dynamical Z-scan measurements, too.

Figure 1 presents the results of the measurement on the sample annealed at $500{ }^{\circ} \mathrm{C}$. The rise time of the CA transient is comparable to the laser pulse duration, which, in this case, is much longer than the electron trapping time of $\sim 100 \mathrm{fs}$ determined from the time-resolved PL measurement. ${ }^{9}$ At the beginning of the OA signal transient, a dip caused by the pump-induced absorption of the probe (TPA) can be clearly distinguished; at later times the dynamics of the OA signal corresponded to the decay of photoinduced absorption from the electron traps. The density of nonequilibrium electronhole pairs excited by the pump beam in the TPA process was of the order of $2 \times 10^{18} \mathrm{~cm}^{-3}$. This concentration was much higher than that of the main electron traps in the As-ionimplanted material, which were determined previously to be single-ionized arsenic antisite defects [approximately 5 $\times 10^{17} \mathrm{~cm}^{-3}$ for GaAs:As annealed at $500^{\circ} \mathrm{C}$ (Ref. 11)]. Therefore, these traps were saturated and the free-electron density decays at a rate determined by the trap emptying time.

The evaluation of this parameter from the measurement data is not straightforward. Even if the ratio CA/OA yields the information on the photoinduced refractive-index change

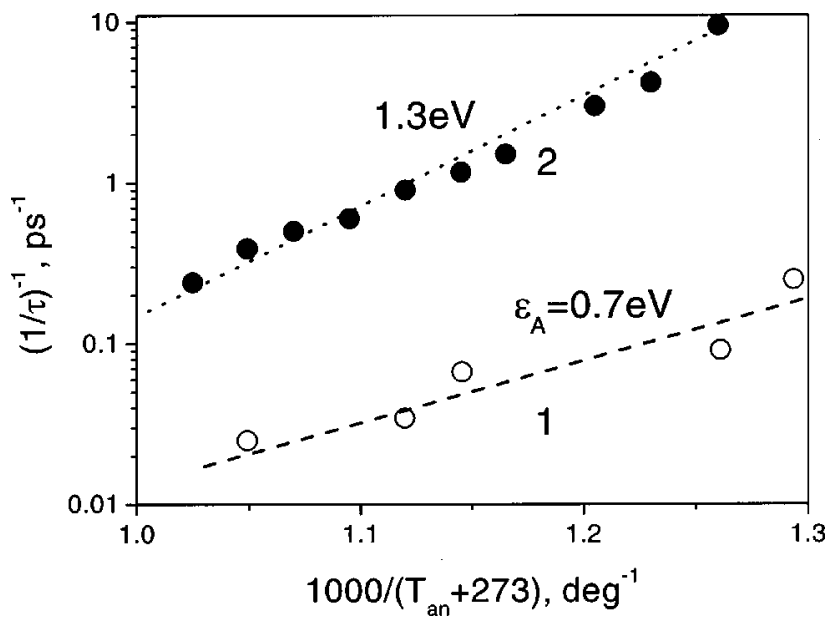

FIG. 2. Recombination (1) and electron trapping (2) rates as a function of inverse anneal temperature.

in the sample, this change could be both due to free-electron and bound-electron contributions. In our experiment, the latter effect can be ignored because its influence is significant during the rising part of the transients only. Therefore, the dynamic refractivity signal will be caused only by the freeelectron-induced lens that will disappear as fast are recombining the electrons excited by the pump beam. Electron diffusion that could lead to a similar effect is taking place at a much longer time scale (a few nanoseconds). Although a simple analytical relation between the nonequilibrium electron density and the measured CA/OA signal does not exist, the numerical simulation of the experiment shows that it is close to a linear one when the sample is placed at 2-5 Rayleigh lengths from the laser beam waist.

Figure 1(b) shows the temporal dependencies of the $\mathrm{CA} / \mathrm{OA}$ ratio for three GaAs:As samples. By interpreting the decay of this ratio as the result of electron recombination, we had determined the recombination times in samples annealed at different temperatures. The recombination time increased from 4 to $40 \mathrm{ps}$ for samples annealed from 500 to $680^{\circ} \mathrm{C}$; the Arrhenius plot of these data is presented on Fig. 2. Activation energy determined from this plot is only a half of the activation energy of $1.3 \mathrm{eV}$ found for the electron trapping times in Ref. 9, which are also presented in Fig. 2 for comparison. Assuming that the electrons are trapped at ionized As antisites $\left(\mathrm{As}_{\mathrm{Ga}}\right)$ and the holes at neutral $\mathrm{As}_{\mathrm{Ga}}$ defects, this difference could be explained by the different anneal dynamics of $\mathrm{As}_{\mathrm{Ga}}$ and their main compensating defects-gallium vacancies.

In principle, the trap emptying or recombination times could be found from the analysis of the photoinduced absorption dynamics that can be seen on the OA signal transients. However, relatively simple dynamics, which is illustrated on Fig. 1(a), can be observed only on OA traces measured for samples annealed at lower temperatures. Figures 3(a) and 3(b) show OA traces measured at different pump pulse intensities on GaAs:As samples annealed at 600 and $700{ }^{\circ} \mathrm{C}$, respectively. The photoinduced absorption that was observed on samples annealed at lower temperatures was replaced by photoinduced transparency. Although this effect was relatively weak for the sample annealed at $600^{\circ} \mathrm{C}$, it dominated the whole transient absorption signal of the 

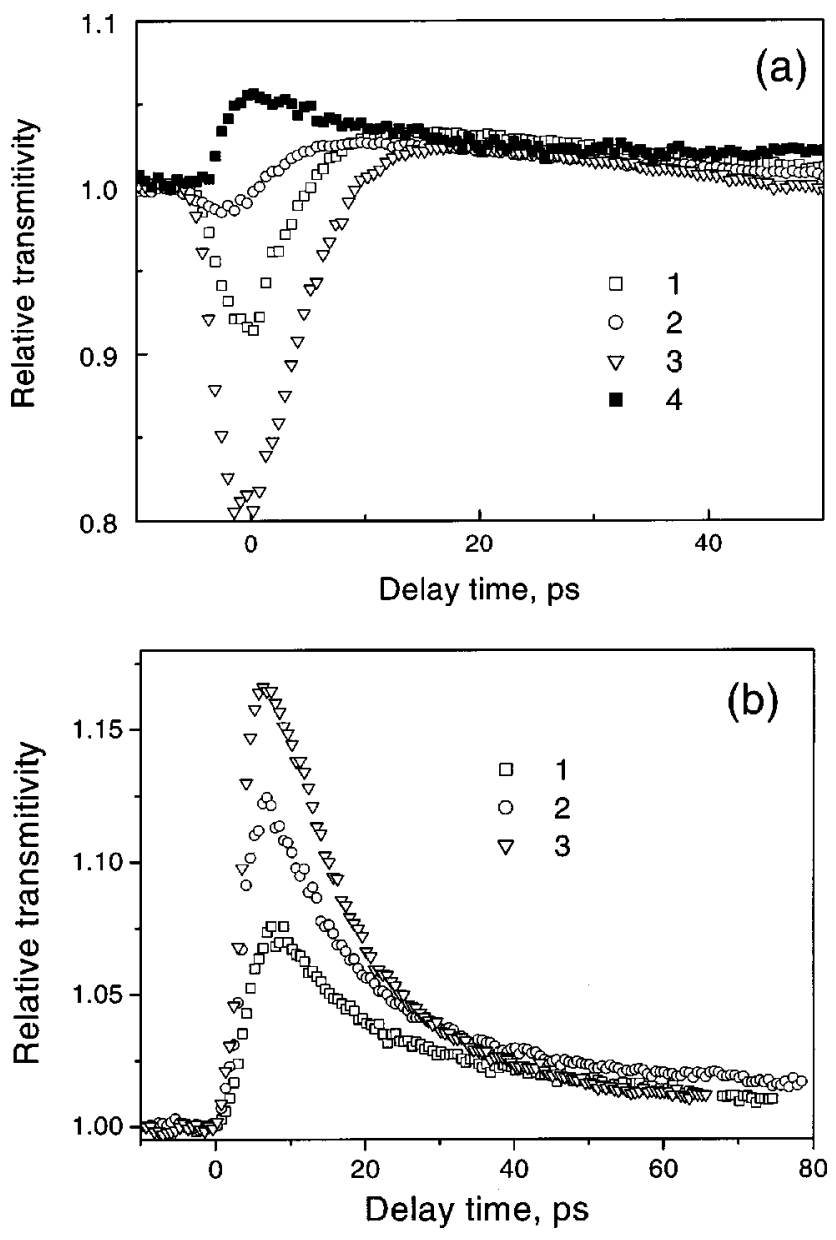

FIG. 3. Transient transmitivity of two GaAs:As samples at different pump pulse energies. (a) Sample annealed at $600{ }^{\circ} \mathrm{C}$. Pump pulse energies were $0.35 \mu \mathrm{J}(1), 1.2 \mu \mathrm{J}(2), 4.7 \mu \mathrm{J}(3)$, and $11 \mu \mathrm{J}(4)$. (b) Sample annealed at $700{ }^{\circ} \mathrm{C}$. Pump pulse energies were $1.5 \mu \mathrm{J}(1), 4.5 \mu \mathrm{J}(2)$, and $10 \mu \mathrm{J}(2)$.

sample annealed at $700{ }^{\circ} \mathrm{C}$ and even covered up the initial cross correlation of the pump and probe pulses due to TPA. The magnitude of the induced transparency signal does not depend on the pump pulse energy for the $600^{\circ} \mathrm{C}$ sample, but for the $700^{\circ} \mathrm{C}$ sample it increased, saturated, and started to decrease with increasing pump energy.

It should be noted that this effect could not be explained by the presence of sole deep defects (arsenic antisites). Pump pulse absorption at these defects would induce the transparency for the probe pulse only as long as the photoexcited electrons are not trapped again. The electron trapping time in the sample with $T_{A}=700{ }^{\circ} \mathrm{C}$ was about $4.5 \mathrm{ps},{ }^{9}$ much shorter than the duration of the observed photoinduced effects. On the other hand, the assumed presence of defects (point or extended) of a second type would qualitatively clarify the majority of the experimental observations. Electrons transferred from those defects will be trapped at ionized $\mathrm{As}_{\mathrm{Ga}}$ centers and, after filling these traps, will establish a nonequilibrium population in the conduction band. The recovery of that population will be determined by the trap emptying, which, in this case, will originate from electrons jumping from the trapping centers back to the defects of origin.

Structural investigations of GaAs:As samples prepared under identical conditions as the ones used in the present investigation ${ }^{11}$ indicated a possible origin of the second type of defects. The results of the transmission electron micrography investigation of As-ion-implanted GaAs were interpreted in terms of As precipitate formation after annealing at temperatures in excess of $600{ }^{\circ} \mathrm{C}$. As it was experimentally demonstrated in Ref. 12, these metallic arsenic precipitates can contribute to a reasonable absorption component out to $1.7 \mu \mathrm{m}$. At the large optical excitations of our experiments, this component would be saturated by the pump pulse and induce the transient transparency for the probe beam. Moreover, the nonexponential decay of the induced refractivity (free electrons) could be also understood when considering the fact that it might be caused by electrons hopping between randomly distributed As-antisite traps and Asprecipitates.

In conclusion, high-energy, long-wavelength laser pulses and a modified pump-and-probe technique were used to investigate the free-electron and trap-filling dynamics in ELO GaAs:As samples annealed at various temperatures. The carrier recombination time was found to be $4 \mathrm{ps}$ in a sample annealed at $500{ }^{\circ} \mathrm{C}$ and increased with annealing temperature. Photoinduced transparency was discovered in samples annealed at high temperatures. This effect, which was explained in terms of absorption at the As precipitates, can be exploited for mode locking of long-wavelength lasers.

This work was supported in part by the EC INCOCOPERNICUS project "DUO-devices for ultrafast optoelectronics" and the Lithuanian Science and Study Foundation.

${ }^{1}$ S. Gupta, M. Y. Frankel, J. A. Valdmanis, J. F. Whitaker, G. A. Mourou, F. W. Smith, and A. R. Calawa, Appl. Phys. Lett. 59, 3276 (1991).

${ }^{2}$ A. Claverie, F. Namavar, and Z. Liliental-Weber, Appl. Phys. Lett. 62, 1271 (1993).

${ }^{3}$ A. Krotkus, S. Marcinkevičius, J. Jasinski, M. Kaminska, H. H. Tan, and C. Jagadish, Appl. Phys. Lett. 66, 3304 (1995).

${ }^{4}$ H. Fujioka, J. Krueger, A. Prasad, X. Liu, E. R. Weber, and A. K. Verma, J. Appl. Phys. 78, 1470 (1995).

${ }^{5}$ F. Ganikhanov, G. R. Lin, W. C. Chen, C. S. Chang, and C. L. Pan, Appl. Phys. Lett. 66, 3465 (1995).

${ }^{6}$ M. J. Lederer, B. Luther-Davies, H. H. Tan, and C. Jagadish, IEEE J. Quantum Electron. 34, 2150 (1998).

${ }^{7}$ P. Grenier and J. F. Whitaker, Appl. Phys. Lett. 70, 1998 (1995).

${ }^{8}$ H. S. Loka, S. D. Benjamin, and P. W. E. Smith, IEEE J. Quantum Electron. 34, 1426 (1998).

${ }^{9}$ S. Marcinkevičius, C. Jagadish, H. H. Tan, M. Kaminska, K. Korona, R. Adomavičius, and A. Krotkus, Appl. Phys. Lett. 76, 1306 (2000).

${ }^{10}$ J. Wang, M. Sheik-Bahae, A. A. Said, D. J. Hagan, and E. W. Van Stryland, J. Opt. Soc. Am. B 11, 1009 (1994).

${ }^{11}$ G. R. Lin, W. C. Chen, C. S. Chang, S. C. Chao, K. H. Wu, T. M. Hsu, W. C. Lee, and C. L. Pan, IEEE J. Quantum Electron. 34, 1740 (1998).

${ }^{12}$ M. R. Melloch, J. M. Woodall, E. S. Harmon, N. Atique, D. D. Nolte, J. C. P. Chang, and N. Otsuka, Semi-insulating III-V Materials. Warsaw, Poland 1994 (World Scientific, Singapore, 1994). 